\title{
Imagery effects on early visual processing
}

\author{
GILLIAN RHODES and ANN O'LEARY \\ Stanford University, Stanford, California
}

\begin{abstract}
Three experiments were carried out to test the hypothesis that visual imagery activates patternprocessing mechanisms in the visual system. This hypothesis may be contrasted with one which posits that the effort required to maintain an image impairs performance on subsequent visual tasks. In Experiment 1, subjects imaged vertical, square-wave gratings confined to one quadrant of the imaginal visual field. This raised thresholds for detecting subsequently presented gratings in all four quadrants, relative to a control condition in which no imaging was required. The orientation specificity of this effect was tested in two further experiments by varying the orientation of adapting and test gratings orthogonally (vertical and horizontal orientations were used). In Experiment 2, a faint grating was present during adaptation to help subjects localize their images and a small fixation bar was scanned to prevent the formation of retinal afterimages. The presence of the faint grating itself produced orientation-specific and location-specific threshold elevation. However, imagining that this grating was of high contrast did not produce any further elevation of the thresholds. In Experiment 3, high-imagery subjects adapted to more easily imaged centrally located gratings. No threshold elevation was found. These results suggest that imagery affects detection thresholds as a result of the effort required rather than by adapting orientation-specific mechanisms in the visual cortex.
\end{abstract}

Imagery and perception often have similar perceptual consequences. Several recent reviews have summarized the evidence for this functional equivalence of imagery and perception in such diverse tasks as stimulus identification, localization, and discrimination (Finke \& Shepard, in press; Shepard, 1978; Shepard \& Podgorny, 1978). These phenomena suggest that imagery and perception use the same information-processing mechanisms in the brain. Finke (1980) has argued that the equivalence extends to early levels of visual processing, where the imagery effects do not depend on subjects' knowledge or expectations. In particular, he has proposed that imagery can directly activate early pattern-processing mechanisms in the visual system. This is the "perceptual equivalence" hypothesis, and it is consistent with early neuropsychologists' views that neural mechanisms underlying imagery are located in the primary areas of the sensory cortex (Beritoff, 1965; Hebb, 1968). The strongest evidence that visual imagery activates early pattern-processing mechanisms comes from imagery-induced McCollough aftereffects (Finke \& Schmidt, 1977, 1978; Kunen \& May, 1980). This orientation-contingent color aftereffect (see McCollough, 1965) has been obtained when vertical and horizontal bars are imagined on real alternating chromatic backgrounds of complementary colors. Finke claims that the effect is due to imagery-induced adaptation of

We thank Ron Finke, Jennifer Freyd, Steven Pinker, Roger Shepard, and Brian Wandell for their helpful advice. We would also like to thank Susan Zare for her programming assistance and Kathleen Wets for helping to test the subjects in Experiment 3. This work was supported by National Science Foundation Grants BNS81-14916 and 82-16546 to Steven Pinker and National Science Foundation Grant BNS 80-05517 to Roger Shepard. Requests for reprints should be sent to G. Rhodes, Psychology Department, Bldg. 420, Jordan Hall, Stanford, CA 94305. orientation-specific mechanisms in the visual cortex, and is therefore evidence for the perceptual equivalence hypothesis.

The reliability of the imagery-induced McCollough effect has, however, come into question. Broerse and Crassini (1980) failed to obtain an imagery-induced McCollough effect, using a pretest/posttest design (color associations were made to the vertical and horizontal gratings before and after imagery induction) to correct for any response bias in color choices. Unlike Finke and Schmidt, they allowed subjects to give "no-color" responses, making their procedure less sensitive.

Finke's interpretation of the effect has also been challenged. Harris (1982) argues that the imagery effect occurs during testing, not during adaptation. On examining Finke and Schmidt's original data, Harris found that if all the data are included, ${ }^{1}$ then vividness of imagery is positively correlated with experiencing any aftereffect (positive or negative), but is not significantly correlated with reporting a negative aftereffect. This suggests that the subjects guessed what color they should see on the test gratings, and that high-imagery subjects imagined that it was actually on the grating. Kaufman, May and Kunen (1981) and Kunen and May (1980) have also suggested that the imagery-induced McCollough effect may depend on mechanisms that are different from those of the perceptual McCollough effect. The imagery effect appears to involve adaptation to higher spatial frequencies in the stimuli, and is reversed if these are removed by blurring the to-be-imagined gratings. The perceptual effect, however, appears to depend on adaptation to the lower spatial frequencies (May \& Matteson, 1976).

Doubts about whether imagery-induced and perceptually induced McCollough aftereffects are mediated by the 
same mechanisms suggest that other tests of the perceptual equivalence hypothesis are necessary. Moreover, even if the imagery-induced McCollough effect is mediated by the same mechanisms as the perceptual effect, it is not clear what these mechanisms are, because the perceptual McCollough effect itself is not well understood (Skowbo, Timney, Gentry, \& Morant, 1975). It certainly does not appear to result from any simple fatiguing of orientation-selective neurons, because it can last for several months (Jones \& Holding, 1975). The long persistence of the McCollough effect suggests that some sort of associative learning is occurring, although this would lead one to expect a positive, rather than a negative, aftereffect. There is probably an initial adaptation of orientation-specific and color-opponent neurons, leading to the negative color aftereffect, which allows the subjects to learn, during the posttest, to associate these colors with the vertical and horizontal bars, producing long-term persistence. This associative-learning hypothesis is consistent with the initial rapid decrease in the size of the aftereffect, which is followed by a much slower rate of decrease (Favreau \& Corballis, 1976). This account of the McCollough effect suggests that the imagery-induced effect might depend on associative learning, not on fatiguing orientation-selective mechanisms in the visual cortex.

To find out whether early pattern-processing mechanisms in the visual system can be activated by imagery, we need to study phenomena that are better understood in the perceptual domain and that are known to depend on the activation of such mechanisms. Orientation-specific threshold elevation is one such phenomenon. Inspection of a grating of a particular orientation is known to raise the threshold for the detection of a grating of the same orientation. This occurs because inspection of the grating fatigues cells in the visual cortex that are tuned to the orientation of that grating (Blakemore \& Campbell, 1969; Pantle \& Sekuler, 1968). We carried out three experiments to investigate the effect of imagery-induced adaptation on detection thresholds. Strict psychophysical procedures were used to measure thresholds for detecting gratings after imaging gratings. The reader should note that, because our procedure required the subject to imagine the stimulus in a designated portion of the visual field, the term "imaging" is used in this paper to refer to imagining a pattern on the display rather than in the "mind's eye."

We were also interested in testing the array theory of mental imagery (Kosslyn, 1980; Kosslyn, Pinker, Smith, \& Schwartz, 1979). This theory is based on the spatial nature of imagery (Finke \& Kosslyn, 1980; Finke \& Kurtzman, 1981; Podgorny \& Shepard, 1978). According to this theory, the structure underlying perceptual and imaginal representations is a two-dimensional array which maps homeomorphically onto the visual field. Such a structure is consistent with the retinotopic organization of the striate cortex (Hubel \& Wiesel, 1979; Luria, 1973), although the array theory itself makes no claims about the neurophysiological substrate of the array. This the- ory predicts that any perceptual effect that is locationspecific should remain so when induced by imagery. Suprathreshold adaptation effects appear to be locationspecific (Blakemore, Muncey, \& Ridley, 1973; Blakemore \& Nachmias, 1971; Blakemore \& Sutton, 1969). If changes in thresholds themselves are similarly locationspecific, then the array theory of mental imagery would predict that imagery-induced threshold elevation should also be location-specific. If imagery activates neurons in the visual cortex that are tuned to particular orientations or spatial frequencies, then it should be possible to fatigue such neurons by imagining a grating of the appropriate orientation or spatial frequency. According to the array theory of mental imagery, this should be restricted to neurons with receptive fields in the part of the visual field in which the grating was imagined.

Threshold elevation following imagery could also occur as a result of the effort required by the imagery task. According to this interpretation, the effort required would lead to impaired performance on the subsequent task, possibly by causing some depletion of the resources needed to create a representation of the test grating that could mediate recognition, or by lowering general motivation levels. The perceptual equivalence and effort hypotheses make different predictions about the location-specificity of imagery-induced threshold elevation. The perceptual equivalence hypothesis predicts that thresholds would be elevated only in the imagery-adapted quadrant, whereas the effort hypothesis predicts that thresholds would be elevated independent of the location of the imaged adaptation grating. The perceptual equivalence hypothesis is clearly the more theoretically interesting of the two, and it was this hypothesis that the present experiments were primarily designed to test. In Experiment 1, we tested the hypothesis that imagery-induced adaptation would produce location-specific threshold elevation for detecting subsequently presented patterns. In Experiments 2 and 3, we tested the orientation specificity of the effect.

\section{EXPERIMENT 1}

\section{Subjects}

Five women and three men were paid for participating in the experiment. All were staff or students at Stanford University.

\section{Stimuli and Apparatus}

The stimuli consisted of gray cards with light and dark gray bars alternating to form a grating in one quadrant. Four such cards were prepared, each containing a low-contrast, 0.63 -cycle/degree (cpd), vertical square-wave grating in one of the four quadrants. These cards were made using Chartpak shading film ( 65 lines of dots per inch and $10 \%, 20 \%$, and $30 \%$ densities of shading). The grating consisted of alternating vertical bars of $10 \%$ and $30 \%$ density shading film, and the remaining three quadrants were covered with $20 \%$ density shading film. Three additional, uniformly gray cards were prepared using $20 \%$ density shading film. Two of these had centrally placed fixation points, one subtending $.32^{\circ}$ of visual angle and the other subtending $.16^{\circ}$ of visual angle; these will be referred to as the "large" and the "small" fixation points, respectively. These stimulus cards were used in the forced-choice discrimina- 
tion sequence described below. The observers were required to discriminate the uniform card from a card containing a grating. Because the average luminance of these two cards was always equal, discriminations could not be based on luminance differences. A white card was also constructed on which there was a cross composed of 15 vertical and 15 horizontal black dots spaced $1 \mathrm{~cm}$ apart. This was used during the adapting foreperiod described below. All cards measured $15 \times 16 \mathrm{~cm}$ and were viewed at an apparent distance of $80 \mathrm{~cm}$ in an Iconix four-field tachistoscope, thus subtending a visual angle of $11.4^{\circ}$. All fields were maximally illuminated (10 fL). The tachistoscope was controlled by a modified Digital Group Z80-SYS5 microprocessor.

\section{Procedure}

Each subject completed five 1.5-h sessions. At the beginning of the first session, Slee's (1980) Visual Elaboration Scale (VES), a test of imagery ability, was administered. Then 25 practice trials were given. There were 120 experimental trials in a session.

Each trial consisted of an imaging foreperiod, followed by a forced-choice discrimination in which the subject decided whether a vertical square-wave grating had appeared in the first or second of two intervals. The appearance of the grating in either of the two intervals was equiprobable, and subjects were told this. The grating was presented randomly in each of the four quadrants, subject to the constraint that it appear equally often in each quadrant within a session. The subjects knew that it was equally likely to appear in any of the four quadrants on each trial. At the end of each session, the subjects estimated the percentage of trials on which they had successfully imaged the adapting grating.

The adapting foreperiod. Each trial began with an adaptation period, during which the subject imaged, in one quadrant, a highcontrast, 0.63 -cpd, vertical square-wave grating. At the beginning of each session, the subject was briefly shown a picture of this striped pattern. The sessions were blocked by the quadrant in which the grating was imaged and were held on different days to minimize any difficulty that subjects might have in changing the locations of their imaged gratings. There was also one session during which no imaging was required during the adaptation period. The order of these five sessions was randomized for each subject.

The card with the dotted cross was shown during the adaptation period, and on imaging trials the subject was instructed to use the horizontal dots to help him/her imagine the grating in the correct phase. The dots, which were spaced $1 / 2$ cycle apart, indicated where each bar of the grating began and ended. Fixation was maintained at the center of the cross. The subject initiated each trial by pressing a key labeled "start." After the dotted cross appeared, the subject formed an image (or relaxed if it was the no-image session) and pressed the "start" key again. This began a timed adaptation period, during which the dotted cross remained visible and the subject tried to maintain an image. The subject was instructed to "refresh" or reconstruct the image, if it faded. The adaptation period lasted for $60 \mathrm{sec}$ on the first trial of a session and for $10 \mathrm{sec}$ on all subsequent trials. Blakemore and Campbell (1969) found that these adaptation periods produced and maintained an optimal level of adaptation to visually presented gratings. On each trial, the adaptation period was followed by the forced-choice discrimination sequence described below. The subject was instructed to stop imaging and to relax as soon as the discrimination sequence began.

The forced-choice discrimination task. A two-alternative forcedchoice procedure was used. The discrimination sequence was as follows: A small fixation point was displayed for $1 \mathrm{sec}$, followed by the first stimulus interval; a large fixation point was displayed for $1 \mathrm{sec}$, followed by the second stimulus interval; and finally the small fixation point reappeared to signal the end of the sequence. A vertical, square-wave grating was presented in one interval, and a uniformly gray card was presented in the other interval. The subject decided whether the grating appeared in the first or second interval, that is, before or after the large fixation point, and responded by pressing one of two keys, labeled "first" and "second." The 120 trials were presented in a different random order (determined by the microprocessor) in each session. The test gratings were presented randomly in the four quadrants, with an equal number occurring in each. The subject was informed that the grating was equally likely to appear in any quadrant and in either interval.

Four interleaved, independent staircases, one for each quadrant, were used during each session. All staircases began at $3 \mathrm{msec}$ exposure for the discrimination stimuli. For each staircase, the exposure time was increased by $.5 \mathrm{msec}$ following an incorrect response and reduced by $.5 \mathrm{msec}$ following two consecutive correct responses (to stimuli in the appropriate quadrant). The turning points of each staircase were averaged to obtain the $71 \%$ correct threshold.

\section{Results and Discussion}

Scores on the VES ranged from 9 to 14 (M $=12$, SD $=1.5$ ). Possible scores on the scale range from 0 to 15 , with higher scores indicating higher imagery ability. Slee (1980) reports that the mean VES score with population samples of 50 or more is within the range 8-9 and the standard deviation is within the range 2.9-4.0. Therefore, all the subjects were average or above in imaging ability. The subjects' estimates of the percentage of trials on which they had an image ranged from $55 \%$ to $100 \%$, with a mean of $89 \%$ and a standard deviation of $12 \%$.

Thresholds were calculated for detecting the square wave in each quadrant for each session. The number of turning points per staircase ranged from 4 to $15(\mathrm{M}=$ 9.4, $\mathrm{SD}=2.0$ ). Detection thresholds were higher for the imaging sessions $(M=2.48 \mathrm{msec})$ than for the noimaging session $(\mathrm{M}=2.28 \mathrm{msec})[\mathrm{t}(7)=2.38, \mathrm{p}<$ .025 , one-tailed]. However, this effect was not locationspecific. The threshold for the quadrant in which the subject had imaged during adaptation $(\mathrm{M}=2.51 \mathrm{msec})$ was not significantly higher than that for the other quadrants $(M=2.47 \mathrm{msec})[\mathrm{t}(7)<1, \mathrm{n} . \mathrm{s}$.]. There were no differences between quadrants with respect to this effect $[\mathrm{F}(3,21)=2.45$, n.s. $]$. Thus, detection thresholds were elevated following imagery, as predicted, but this was not location-specific. This is consistent with the effort hypothesis, which predicts that the effort required by the imagery task causes a generalized reduction of performance for subsequent detection. All subjects reported that the imagery task was difficult.

In the no-imagery condition, the gratings were not equally detectable in the four quadrants. Mean thresholds, in milliseconds, for the quadrants were: 2.01 (upper right), 2.25 (lower right), 2.65 (lower left), and 2.19 (upper left). Post hoc Scheffé comparisons showed that detection was significantly worse in the lower left quadrant than in the other quadrants $[F(1,21)=9.35, p<.05]$. No other pairwise differences were significant. This nonhomogeneity should be interpreted with caution, since the stimuli were hand-made and there may have been subtle differences between the stimuli for the four quadrants. It was not replicated in Experiment 2.

\section{EXPERIMENT 2}

It is possible that adaptation of pattern-processing mechanisms in the cortex could produce the non-location- 
specific threshold changes found in Experiment 1, if the adapted neurons were not selective for position. This would be the case if complex cells were adapted. Although these cells are not selective for position, they are selective for orientation, and so adapting them should produce orientation-specific threshold elevation. Experiment 1 did not test whether the adaptation was orientation-specific. Experiment 2 was designed to do so. If threshold elevation was not orientation-specific, then the effort hypothesis would be supported. A nonvisual, effortful task, counting backwards in threes, was also included in Experiment 2 . If threshold elevation was non-orientation-specific but nevertheless did not occur in the counting condition, this would indicate that resources specific to visual tasks were depleted by the imaging task. If thresholds were elevated in this condition, it would indicate that early visual processing can be affected by a preceding effortful task which does not engage visual mechanisms. This would be more consistent with the possibility that a difficult imagery task lowers general motivation levels.

Subjects in Experiment 1 had reported difficulty in localizing the grating properly. It was therefore possible that the non-location-specific threshold elevation obtained in Experiment 1 had resulted from failure to keep the images entirely in one quadrant. In Experiment 2, a lowcontrast version of the adapting grating was presented during adaptation and the subjects were asked to imagine that it was high-contrast. The presence of the faint grating was intended to ensure that the subjects' images were, in fact, localized in one quadrant of the display.

\section{Subjects}

\section{Method}

Five women and three men were paid for their participation in the experiment. The subjects were not preselected for imagery ability. However, all obtained average or above-average scores on the VES (scores ranged from 9 to $13 ; M=10.5, S D=1.9$ ).

\section{Stimuli and Apparatus}

The stimuli were similar to those used in Experiment 1, except that the spatial frequency of the gratings was $1.26 \mathrm{cpd}$ and the display size was $12 \times 12 \mathrm{~cm}$. The adapting card with the dotted cross was not used. Instead, the adapting cards each contained a lowcontrast, vertical or horizontal, 1.26-cpd square-wave grating. The subjects imagined this low-contrast grating to be high-contrast (i.e., black and white). Both horizontal and vertical gratings were used. The low-contrast adapting gratings were constructed by placing 0.5 $\mathrm{cm}$-wide strips of $10 \%$ density shading every $1 \mathrm{~cm}$ in one of the four quadrants. Each adapting card also contained a small, faint cross $(4 \times 4 \mathrm{~mm})$ in the quadrant diagonally across from that containing the grating. In the imaging conditions, the subjects had to imagine that both this cross and the grating were of high contrast. This was intended to help the subjects keep the imaged high-contrast grating localized in one quadrant only, by providing a reference point or anchor in another part of the field. Each adapting card had a 1-cm-long (one cycle) black fixation bar centered in the middle of the card. The fixation bar was perpendicular to the grating. The subjects scanned this bar while imaging, so that the faint adapting grating would not produce a retinal afterimage.

\section{Procedure}

The general procedure was the same as that of Experiment 1, with each trial consisting of an adaptation period followed by a forced-choice discrimination task. Again, 25 practice trials preceded the 120 experimental trials. Each subject completed four 1-h sessions, which were blocked by adaptation condition. Two of these were imaging conditions. In one, the adaptation and test gratings had the same orientation (Condition S); in the other, they were perpendicular to each other (Condition D). In these imaging sessions, ( $S$ and $D$ ), during the adaptation phase of each trial, the subjects imagined that the faint grating and the small cross in the diagonally opposite quadrant were high-contrast. The subjects were instructed to move their eyes back and forth along the fixation bar while the adapting card was visible. The third adaptation condition was a noimaging control condition ( $\mathrm{N}$ ), in which the subjects relaxed and scanned the fixation bar during the adaptation period. The fourth was a counting control condition (C), in which, during the adaptation period, subjects counted backwards in multiples of three (from a number given by the experimenter) as quickly and accurately as possible.

Each subject adapted to gratings in one quadrant only (two subjects for each quadrant). The faint grating was present in the control conditions ( $\mathrm{N}$ and $\mathrm{C}$ ) and, during the adaptation foreperiod, was always in the same orientation as it was in the test. Half the subjects adapted to vertical gratings, and half adapted to horizontal gratings. For half the subjects, the $71 \%$ threshold for detecting gratings of the same orientation as the adaptation grating (S) was determined before that for gratings perpendicular to the adaptation grating (D). This order was reversed for the rest of the subjects. This factor and the adaptation orientation were counterbalanced. The $\mathrm{N}$ and $\mathrm{C}$ sessions were interspersed randomly with the $\mathrm{S}$ and $\mathrm{D}$ sessions. The location (quadrant) in which the test grating appeared on each trial was random, subject to the constraint that all four quadrants be tested equally often within each session. Thus, in each session the adaptation and test gratings occurred in the same quadrant on $25 \%$ of the trials (30) and in different quadrants on $75 \%$ of the trials $(90)$.

At the end of each imaging session, the subjects rated how much effort the imaging had required and how successful they had been. After the $\mathrm{C}$ session, they rated how much effort the counting had required. All ratings were made on 7-point scales. A higher rating indicated greater effort or success.

\section{Results and Discussion}

The number of turning points in each staircase ranged from 4 to $15(M=9.1, S D=2.1)$. Mean detection thresholds for each condition (S, D, N, and C) are shown in Table 1. The results for thresholds in the adapted quadrant and those in other quadrants are presented separately, in order to examine the location specificity of the imaging effects. In all conditions in which the orientation of the adaptation and test gratings was the same (S, N, and C), detection thresholds for gratings presented in the same quadrant as the adaptation grating were elevated relative to those presented in a different quadrant. No elevation occurred when the test gratings were perpendicular to the adaptation grating (D). This indicates that the low-contrast adaptation grating itself produced orientation-specific and location-specific elevation of detection thresholds. Imagining that the adaptation grating was high-contrast did not further elevate the thresholds.

These results were confirmed when the data were subjected to a two-way randomized-blocks analysis of variance. The main effects of adaptation condition and location of test gratings (same as or different from the adaptation quadrant) were both significant $[\mathrm{F}(3,49)=$ $3.96, \mathrm{p}<.05$, and $\mathrm{F}(1,49)=33.04, \mathrm{p} \ll .01$, respec- 
Table 1

Mean Detection Thresholds (in Milliseconds) in Experiment 2

\begin{tabular}{|c|c|c|c|c|c|c|c|c|}
\hline \multirow{3}{*}{$\begin{array}{l}\text { Location of } \\
\text { Test Grating }\end{array}$} & \multicolumn{8}{|c|}{ Adapting Condition } \\
\hline & \multicolumn{2}{|c|}{$\begin{array}{c}\text { Same Image and } \\
\text { Test Orientation (S) }\end{array}$} & \multicolumn{2}{|c|}{$\begin{array}{c}\text { Perpendicular Image } \\
\text { and Test Orientation (D) }\end{array}$} & \multicolumn{2}{|c|}{ No Image (N) } & \multicolumn{2}{|c|}{ Count (C) } \\
\hline & Mean & SE & Mean & SE & Mean & $\mathrm{SE}$ & Mean & SE \\
\hline Same as imaged grating & 3.6 & 0.3 & 2.9 & 0.2 & 4.0 & 0.4 & 3.5 & 0.3 \\
\hline Different from imaged grating & 2.6 & 0.2 & 2.9 & 0.3 & 2.9 & 0.2 & 2.8 & 0.2 \\
\hline
\end{tabular}

tively]. The interaction was also significant $[F(3,49)=$ 4.25 , $\mathrm{p}<.01$ ], and so tests of simple main effects were carried out. There was a significant location-specific elevation of the detection thresholds in all the conditions in which the test and adaptation gratings had the same orientation $[\mathrm{F}(1,49)=18.58, \mathrm{p} \ll .01(\mathrm{~S}), \mathrm{F}(1,49)=19.79$, $\mathrm{p} \ll .01(\mathrm{~N})$, and $\mathrm{F}(1,49)=7.33, \mathrm{p}<.01)(\mathrm{C})]$, but not in the session in which they were perpendicular to each other, $[F(1,49)=0.00$, n.s. (D)]. Thus, whether the subject imaged during the adaptation period or not, the thresholds for gratings presented in the same quadrant as the adaptation grating were elevated relative to those for gratings presented in the other quadrants only if the faint grating present during this period was in the same orientation as the test gratings $(\mathrm{S}, \mathrm{N}$, and $\mathrm{C})$. When it was perpendicular to the test grating (D), no threshold elevation was observed. This pattern of results suggests that the adaptation grating itself produced location-specific and orientation-specific elevation of detection thresholds.

Separate comparisons were also carried out between conditions ( $\mathrm{S}, \mathrm{D}, \mathrm{N}$, and $\mathrm{C}$ ) when the test and adaptation stimuli were in the same or different quadrants. When they were in the same quadrant, there was a significant difference between thresholds in the four adaptation conditions $[F(3,49)=7.38, p \ll .01]$, but when they were in different quadrants, there was no difference between the conditions $[\mathrm{F}(3,49)=0.79$, n.s. $]$. Thus, thresholds depended on the adaptation condition only when the grating was in the same quadrant as the adaptation grating. Pairwise post hoc Scheffé comparisons showed that, in this case, the $\mathrm{S}$ and $\mathrm{N}$ means differed significantly from the $\mathrm{D}$ mean $[\mathrm{F}(3,49)=9.38, \mathrm{p}<.05$, and $\mathrm{F}(3,49)=$ $21.28, \mathrm{p}<.001$, respectively]. The $\mathrm{C}$ mean did not differ significantly from the D mean $[F(3,49)=6.41$, n.s. $]$. Nor did the $S, N$, and $C$ means differ from each other $[F(3,49)$ $=2.4$, n.s. (S and $\mathrm{N}), \mathrm{F}(3,49)=4.34$, n.s. $(\mathrm{N}$ and $\mathrm{C})$, and $F(3,49)=0.28$, n.s. (S and $C)]$.

The gratings were equally detectable in all four quadrants $[\mathrm{F}(3,21)=1.26, \mathrm{n} . \mathrm{s}$.$] . The mean thresholds were$ 2.72 (upper right), 3.41 (lower right), 3.69 (lower left), and 3.31 (upper left). Thus, the nonhomogeneity found in Experiment 1 was not replicated.

The mean rating of effort required by the imagery task was 5.6 on a 7-point scale (SD $=1.0)$. Thus, the imagery task was judged to be fairly difficult. The subjects rated themselves as moderately successful at it $(\mathrm{M}=3.4, \mathrm{SD}$ $=0.7$ ). The mean rating of effort required by the counting task was $3.5(\mathrm{SD}=1.7)$.
To our knowledge, this is the first demonstration of a location-specific adaptation effect on detection thresholds, although location-specific aftereffects have been demonstrated at suprathreshold levels (Blakemore et al., 1973; Blakemore \& Nachmias, 1971; Blakemore \& Sutton, 1969). It is unlikely that this effect is due simply to the formation of retinal afterimages, because subjects scanned a fixation bar during adaptation. However, to rule out this possibility completely would require a different technique from that used in the present experiment, such as a demonstration that the threshold elevation transferred interocularly.

The adaptation grating raised thresholds by $1.0 \mathrm{msec}$ in the $\mathrm{S}$ condition, by $1.1 \mathrm{msec}$ in the $\mathrm{N}$ condition, and by $.7 \mathrm{msec}$ in the $C$ condition. These elevations are much larger than those found in Experiment 1 (where imagery raised thresholds by $.2 \mathrm{msec}$ ), and may have masked any change in thresholds produced by imagery. Therefore, the results do not bear on the question of whether imagery can adapt early visual processing mechanisms.

\section{EXPERIMENT 3}

Because imagery effects may have been masked by adaptation produced by the faint adaptation grating in Experiment 2 , a third experiment was carried out in which the adaptation grating was no longer used. Furthermore, in order to maximize the chances of finding an adaptation effect, subjects were preselected to have vivid imagery.

Three of the eight subjects in Experiment 1 had spontaneously remarked that restricting the image to one quadrant was especially difficult. Even with the faint adaptation grating present in Experiment 2 to help localize the image, the subjects rated the imaging task as difficult. In Experiment 3, all gratings were positioned centrally, making the imaging task easier. Evidence that images are easier to maintain when located centrally comes from work by Finke and Kosslyn (1980) and Finke and Kurtzman (1981). They found that resolution of visual images falls off with distance from the center of the imaginal field, which suggests that more peripheral images (at least those requiring some degree of spatial resolution such as the gratings imaged here) would be harder to maintain. When describing difficulties they had experienced in imaging, two subjects in Experiment 1 noted that the peripheral part of the image was especially difficult to maintain. If the perceptual equivalence hypothesis is correct, then Experi- 
ment 3 should provide optimal conditions for demonstrating imagery-induced threshold elevation. Subjects were vivid imagers and a relatively easy (compared with Experiments 1 and 2) imagery task was used. The orientation specificity of any threshold elevation following imagery was also tested.

Method
Subjects
Sixteen Stanford undergraduates from an introductory psychol-
ogy course participated in the experiment. The subjects were selected
on the basis of having vivid visual imagery. A criterion score of
12 or above (out of 15$)$ on the VES was adopted. The mean VES
score was $13.6(\mathrm{SD}=1.1)$.

\section{Stimuli and Apparatus}

The stimuli were the same as in Experiment 1, except that the gratings appeared in the center of the field rather than in one quadrant. Also, both horizontal and vertical gratings were used, so that the orientation of the imaged and test gratings could be varied orthogonally. Two adaptation cards were constructed, on which the subjects imaged the gratings. One card had eight small black dots, spaced $1 \mathrm{~cm}$ apart, along the top and along the bottom. The dots were half a cycle (one bar width) apart. These were used by the subjects to help them position the vertical grating correctly. A similar card, with eight dots on the left edge and eight along the right edge, was used to position the horizontal grating correctly during the horizontal imaging trials. Both cards had a small black fixation point in the center, on which subjects fixated while imaging the grating. Field intensities were lowered from maximal illumination (10 fL) to low illumination $(2 \mathrm{fL}),{ }^{2}$ to reduce the likelihood that the subjects would go below the minimum exposure time $(.5 \mathrm{msec})$ in staircases. $^{3}$

\section{Procedure}

Detection thresholds were obtained in three different conditions. These were the same as the S, D, and N conditions in Experiment 2. Separate staircases were run in each of these conditions. Staircases began at a 4-msec exposure duration. All subjects were tested in all three conditions. The two imaging conditions (S and D) were administered in one session, and the no-imaging condition $(N)$ was administered in a different session on a different day. At the beginning of each session, there were 20 practice trials. The subjects were not required to image on the practice trials preceding the imaging blocks. Each session lasted approximately $1 \mathrm{~h}$. Half the subjects did the imaging session first, and half did the no-imaging session first. In the imaging session, half the subjects were tested with gratings of the same orientation as their imaged gratings first ( $\mathrm{S}$ and D) and half were tested with gratings of the opposite orientation first (D then $S$ ). Each subject imaged either vertical or horizontal gratings in the two imaging conditions (S and D). Half imaged vertical gratings, and half imaged horizontal gratings. In the no-imaging condition $(\mathrm{N})$, half the subjects were tested on vertical gratings and half were tested on horizontal gratings. The four factors-order of sessions (imaging and no imaging), order of imagery conditions within the imagery session (SD or DS), orientation of imaged gratings (horizontal or vertical), and orientation of test gratings in the no-imagery condition (horizontal or vertical)-were counterbalanced $(2 \times 2 \times 2 \times 2)$ to produce 16 different treatments. Each subject was assigned randomly to one of these treatments.

The adaptation foreperiod and discrimination sequence were exactly as in Experiment 1 . The only difference between the two experiments was that in Experiment 3 the gratings were presented centrally, rather than in particular quadrants. The imagery instructions were the same as in Experiment 1, and at the beginning of the imagery session each subject was shown the grating he or she was to image. One block of $\mathbf{4 0}$ trials was presented for each condition
(S, D, and N), and separate thresholds were obtained for each. Thus, each subject had a total of 120 trials, 80 in one session and 40 in the other. At the end of the imaging session, the subjects were asked to describe any difficulties they had experienced with the imaging task and to estimate the percentage of trials on which they had successfully imaged.

\section{Results and Discussion}

The number of turning points in each staircase ranged from 4 to $15(M=8.6, S D=2.1)$. Detection thresholds did not differ in the three conditions, $S(M=4.18)$, $\mathrm{D}(\mathrm{M}=4.40)$ and $\mathrm{N}(\mathrm{M}=4.37)[\mathrm{F}(2,30)=.493$, n.s. $]$. Thus, imaging gratings, either of the same orientation as the test gratings or perpendicular to them, had no effect on detection thresholds.

The mean percentage of trials on which subjects imaged successfully was $81 \%$ (SD $=12 \%$ ), which was not higher than in Experiment $1(M=89 \%)$. However, the subjects reported little difficulty with the imagery taskeight reported no difficulties at all and eight reported minor difficulties. In Experiment 1, all subjects spontaneously complained about the difficulty of the imaging task. Similarly, in Experiment 2, difficulty was rated to be high ( 5.6 on a 7-point scale). Therefore, it seems that the imaging task was, in fact, easier in Experiment 3 than it was in Experiments 1 and 2. The absence of an imagery effect on detection thresholds in Experiment 3, which required a relatively easy imaging task for vivid imagers, is more consistent with the effort hypothesis than with the perceptual equivalence hypothesis.

\section{CONCLUSIONS}

The hypothesis that functional equivalence of perception and imagery results from activation of perceptual processing mechanisms during imagery is theoretically attractive. It provides a plausible mechanism for the subjective similarity of images and percepts, and for their functional equivalence in many experimental tasks. The best evidence that this activation extends to early patternprocessing mechanisms in the visual cortex is the imageryinduced McCollough aftereffect. However, neither the reliability nor the interpretation of this effect is clearly established.

To determine whether imagery activates early patternprocessing mechanisms, we investigated the effects of imagery-induced adaptation on thresholds for detecting subsequently presented square-wave gratings. The results from the three experiments reported here failed to support the hypothesis that imagery activates location-specific or orientation-specific mechanisms of the sort found in the primary visual cortex. They are therefore consistent with the results of early studies which failed to find imagery-induced masking of lines (Over \& Broerse, 1972) or imagery-induced tilt aftereffects (Singer \& Sheehan, 1965).

Rather, the results suggest that if imagery elevates detection thresholds, it may be because it requires effort. This conclusion must be regarded as tentative, until ef- 
fort is explicitly manipulated in a single experiment. The mechanism underlying an effort effect might be a depletion of resources needed to create representations that can subserve detection, or some more general lowering of motivation levels.

The main conclusion to be drawn from the results is that pattern-processing mechanisms of the sort found in the primary visual processing areas of the cortex (e.g., location-specific, orientation-specific, width-specific mechanisms) do not appear to be activated during visual imagery. Thus, internally generated images must be represented at higher levels of the visual system than the primary visual cortex. This is consistent with the observation that electrical stimulation of the primary visual cortex produces elementary sensations, such as flashes of light and colored spots, whereas stimulation of the secondary areas produces recognizable images of objects and familiar people (Luria, 1973).

\section{REFERENCES}

BERITOFF, J. S. (1965). Neural mechanisms of higher vertebrate behavior (W. T. Liberson, Ed. and Trans.). Boston: Little, Brown.

Blakemore, C., \& Campbell, F. W. (1969). On the existence of neurones in the visual system selectively sensitive to the orientation and size of retinal images. Journal of Physiology, 203, 237-260.

Blakemore, C., Muncey, J. P. L., \& Ridley, R. M. (1973). Stimulus specificity in the human visual system. Vision Research, 13, 1915-1931.

Blakemore, C. B., \& Nachmias, J. (1971). The orientational specificity of two visual aftereffects. Journal of Physiology (London), 213, 157-174.

Blakemore, C., \& Sutton, P. (1969). Size adaptation: A new aftereffect. Science, 166, 245-247.

BrINDLEY, G. S. (1971). Physiology of the retina and visual pathway. London: Camelot Press.

Broerse, J., \& Crassini, B. (1980). The influence of imagery ability on color aftereffects produced by physically present and imagined induction stimuli. Perception \& Psychophysics, 28, 560-568.

CoRnsweet, T. N. (1970). Visual perception. New York: Academic Press.

Favreau, O. E., \& Corballis, M. C. (1976). Negative aftereffects in visual perception. Scientific American, 235(6), 42-48.

FINKE, R. A. (1980). Levels of equivalence in imagery and perception. Psychological Review, 87, 113-132.

FinKE, R. A., \& Kosslyn, S. M. (1980). Mental imagery acuity in the peripheral visual field. Joumal of Experimental Psychology: Human Perception and Performance, 6, 126-139.

Finke, R. A., \& KurTZMan, H. S. (1981). Mapping the visual field in mental imagery. Joumal of Experimental Psychology: General, 110, 501-517.

FINKE, R. A., \& SchMid, M. J. (1977). Orientation-specific color aftereffects following imagination. Journal of Experimental Psychology: Human Perception and Performance, 3, 599-606.

FINKE, R. A., \& SCHMIDT, M. J. (1978). The quantitative measure of pattern representation in images using orientation-specific color aftereffects. Perception \& Psychophysics, 23, 515-520.

FINKE, R. A., \& SHEPARD, R. N. (in press). Visual functions of mental imagery. In L. Kaufman \& J. Thomas (Eds.), Handbook of perception and human performance. New York: Wiley.
HARRIs, J. P. (1982). The VVIQ and imagery-induced McCollough effects. Perception \& Psychophysics, 32, 290-292.

Hebb, D. O. (1968), Concerning imagery. Psychological Review, 75 , 466-477.

Hubel, H. H., \& WiESEL, T. N. (1979). Brain mechanisms of vision. Scientific American, 241(3), 150-162.

Jones, P. D., \& Holding, D. H. (1975). Extremely long-term persistence of the McCollough effect. Journal of Experimental Psychology: Human Perception and Performance, 1, 323-327.

Kaufman, J. H., May, J. G., \& KUnEN, S. (1981). Interocular transfer of orientation-contingent color aftereffects with external and internal adaptation. Perception \& Psychophysics, 30, 547-551.

KossLYN, S. M. (1980). Image and mind. Cambridge, Massachussetts: Harvard University Press.

Kosslyn, S. M., Pinker, S., Smith, G. E., \& Schwartz, S. P. (1979). On the demystification of mental imagery. The Brain and Behavioral Sciences, 2, 570-581.

KunEN, S., \& MAY, J. G. (1980). Spatial frequency content of visual imagery. Perception \& Psychophysics, 28, 555-559.

Luria, A. R. (1973). The working brain. New York: Penguin Books. MAY, J. G., \& MATTESON, H. H. (1976). Spatial frequency-contingent color aftereffects. Science, 194, 145-147.

McCollough, C. (1965). Color adaptation of edge detectors in the human visual system. Science, 149, 1115-1116.

OVER, R., \& Broerse, B. (1972). Imagined lines fail to induce contour masking. Psychonomic Science, 29, 203-204.

Pantle, A., \& Sekuler, R. (1968). Size detecting mechanisms in human vision. Science, 162, 1146-1148.

PoDGorNy, P., \& SHEPARD, R. N. (1978). Functional representations common to visual perception and imagination. Joumal of Experimental Psychology: Human Perception and Performance, 4, 21-35.

ShePARD, R. N. (1978). The mental image. American Psychologist, 33, 123-137.

ShePard, R. N., \& Podgorny, P. (1978). Cognitive processes that resemble perceptual processes. In W. K. Estes (Ed.), Handbook of learning and cognitive processes (Vol. 5). Hillsdale, NJ: Erlbaum.

Singer, G., Sheehan, P. W. (1965). The effects of demand characteristics on figural after-effect with real and imagined inducing figures. American Joumal of Psychology, 78, 96-101.

Skowbo, D., Timney, B. N., Gentry, T. A., \& Morant, R. B. (1975). McCollough effects: Experimental findings and theoretical accounts. Psychological Bulletin, 82, 497-510.

SLEE, J. A. (1980). Individual differences in visual imagery ability and the retrieval of visual appearances. Journal of Mental Imagery, 4, 93-113.

\section{NOTES}

1. Finke and Schmidt excluded data from subjects who reported using an "associative strategy" (deliberate association of orientation and color) on some or all of the test trials. Almost half the subjects were excluded by this criterion.

2. Bloch's law states that, for brief stimuli (less than $30 \mathrm{msec}$ if the observer is adapted to a moderate illumination level), the product of duration and intensity determines what is seen (Brindley, 1971; Cornsweet, 1970). In other words, for brief stimuli, there is perfect temporal summation. Therefore, it is unlikely that any differences in imagery effects in Experiments 1 and 3 would result from this procedural change.

3. This happened once with two subjects and four times with another subject in Experiment 1.

(Manuscript received May 29, 1984; revision accepted for publication February 26, 1985.) 